placebo group received subsequent life-extending therapy at the time of data cutoff. At that time, of the 251 patients in the placebo group who had not received subsequent life-extending therapy, $24 \%$ continued to receive placebo, $19 \%$ received other therapies, and $43 \%$ died before receiving any subsequent therapy. We would suggest that the timing of chemotherapy in such patients has not been shown to affect survival and that the post-progression treatment experience of our study patients reflects the standard of care.

Tomasz M. Beer, M.D.

Oregon Health and Science University

Portland, OR

beert@ohsu.edu

\section{Bertrand Tombal, M.D.}

Cliniques Universitaires Saint-Luc Brussels, Belgium

Since publication of their article, the authors report no further potential conflict of interest.

1. Sweeney C, Chen Y-H, Carducci MA, et al. Impact on overall survival (OS) with chemohormonal therapy versus hormonal therapy for hormone-sensitive newly diagnosed metastatic prostate cancer (mPrCa): an ECOG-led phase III randomized trial. J Clin Oncol 2014;32:Suppl:LBA2. abstract.

2. Gravis G, Fizazi K, Joly F, et al. Androgen-deprivation therapy alone or with docetaxel in non-castrate metastatic prostate cancer (GETUG-AFU 15): a randomised, open-label, phase 3 trial. Lancet Oncol 2013;14:149-58.

DOI: $10.1056 /$ NEJMc1410239

\title{
Brain Abscess
}

TO THE EDITOR: In their review article, Brouwer et al. (July 31 issue) ${ }^{1}$ suggest the use of vancomycin for the treatment of brain abscesses caused by methicillin-resistant Staphylococcus aureus (MRSA). However, regarding this choice, there are some concerns that should be considered.

First, vancomycin has poor cerebrospinal fluid (CSF) penetration when administered at a standard dose; thus, larger doses should be administered to achieve adequate CSF levels. However, high vancomycin serum concentrations are independently associated with nephrotoxicity. ${ }^{2}$ Second, dexamethasone, used for the management of brain edema, can substantially reduce the penetration of vancomycin into CSF. ${ }^{3}$ Third, infections caused by MRSA strains with high minimum inhibitory concentrations of vancomycin have been associated with increased treatment failure and mortality when vancomycin is used empirically. ${ }^{4}$

Here, we pose the question of whether it is time to review the current approach to antibiotic therapy. We believe that, as first-line empirical treatment for brain abscesses caused by MRSA, newer antibiotic agents, such as linezolid, that achieve higher CSF levels than those observed with vancomycin should be used. 5 Although interindividual variability has been reported, an increasing number of reports have shown the efficacy of linezolid for the treatment of brain abscesses.

Sebastiano Leone, M.D.

Guglielmo Migliorino, M.D.

Andrea Gori, M.D.

San Gerardo Hospital

Monza, Italy

sebastianoleone@yahoo.it
Dr. Leone reports having received educational grants from Pfizer. No other potential conflict of interest relevant to this letter was reported.

1. Brouwer MC, Tunkel AR, McKhann GM II, van de Beek D. Brain abscess. N Engl J Med 2014;371:447-56.

2. van Hal SJ, Paterson DL, Lodise TP. Systematic review and meta-analysis of vancomycin-induced nephrotoxicity associated with dosing schedules that maintain troughs between 15 and 20 milligrams per liter. Antimicrob Agents Chemother 2013;57: 734-44.

3. van de Beek D, Brouwer MC, Thwaites GE, Tunkel AR. Advances in treatment of bacterial meningitis. Lancet 2012;380: 1693-702.

4. Ippolito G, Leone S, Lauria FN, Nicastri E, Wenzel RP. Methicillin-resistant Staphylococcus aureus: the superbug. Int J Infect Dis 2010;14:Suppl 4:S7-S11.

5. Myrianthefs P, Markantonis SL, Vlachos K, et al. Serum and cerebrospinal fluid concentrations of linezolid in neurosurgical patients. Antimicrob Agents Chemother 2006;50:3971-6.

DOI: $10.1056 / N E J M c 1410501$

TO THE EDITOR: In their review article, Brouwer and colleagues recommend the standard fourdrug regimen (isoniazid, rifampin, pyrazinamide, and ethambutol) for the treatment of brain abscesses due to Mycobacterium tuberculosis. This recommendation is in accordance with current guidelines $^{1}$; nevertheless, it must be noted that although this regimen has been studied extensively in patients with respiratory tuberculosis, there is a scarcity of data regarding its use for the treatment of brain abscesses.

Pharmacokinetic studies ${ }^{2}$ have shown that the CSF penetration of ethambutol is poor, with negligible concentrations in patients without meningitis. In contrast, fluoroquinolones, which have good activity against M. tuberculosis, reach CSF levels well above the minimum inhibitory con- 
centrations, ${ }^{3,4}$ although an unequivocal benefit in tuberculous meningitis has not been proved. ${ }^{5}$

Available pharmacokinetic data should induce some caution in recommending the use of ethambutol for the treatment of tuberculosis of the central nervous system and should discourage the use of low doses of ethambutol (15 mg per kilogram of body weight). However, the use of fluoroquinolones in combination with isoniazid, rifampin, and pyrazinamide may deserve consideration in the treatment of this severe condition.

Marco Rizzi, M.D.

Diego Ripamonti, M.D.

Ospedale Papa Giovanni XXIII

Bergamo, Italy

mrizzi@hpg23.it

No potential conflict of interest relevant to this letter was reported.

1. Treatment of tuberculosis: guidelines. 4 th ed. Geneva: World Health Organization, 2009.

2. Donald PR. Cerebrospinal fluid concentrations of antituberculosis agents in adults and children. Tuberculosis (Edinb) 2010; 90:279-92.

3. Pea F, Pavan F, Nascimben E, et al. Levofloxacin disposition in cerebrospinal fluid in patients with external ventriculostomy. Antimicrob Agents Chemother 2003;47:3104-8.

4. Alffenaar JWC, van Altena R, Bökkerink HJ, et al. Pharmacokinetics of moxifloxacin in cerebrospinal fluid and plasma in patients with tuberculous meningitis. Clin Infect Dis 2009;49:1080-2. 5. Ruslami R, Ganiem AR, Dian S, et al. Intensified regimen containing rifampicin and moxifloxacin for tuberculous meningitis: an open-label, randomised controlled phase 2 trial. Lancet Infect Dis 2013;13:27-35.

DOI: 10.1056/NEJMc1410501

TO THE EDITOR: The article by Brouwer et al. mentions several causes of brain abscesses but does not include paracoccidioidomycosis. This fungal disease is the most common systemic endemic mycosis in Central America and South America (but with increasing incidence in Europe and North America) and is the eighth highest cause of death (1.45 deaths per 1 million inhabitants per year) from chronic infectious disease in Brazil. ${ }^{1,2}$

The prevalence of central nervous system involvement in paracoccidioidomycosis (neuroparacoccidioidomycosis) is approximately $10 \%$ and can reach $36 \%$ with more aggressive use of neuroimaging. ${ }^{3}$ However, it is frequently underrecognized and underreported. Neuroparacoccidioidomycosis presents as brain lesions (abscess, granuloma, nodule, or cyst) in $90 \%$ of cases and as meningitis in $10 \%$, is associated with a striking mortality of $44 \%$, and causes substantial sequelae in approximately half the survivors. ${ }^{3,4}$ Consequently, this fungal disease should be considered in patients with brain abscess, especially
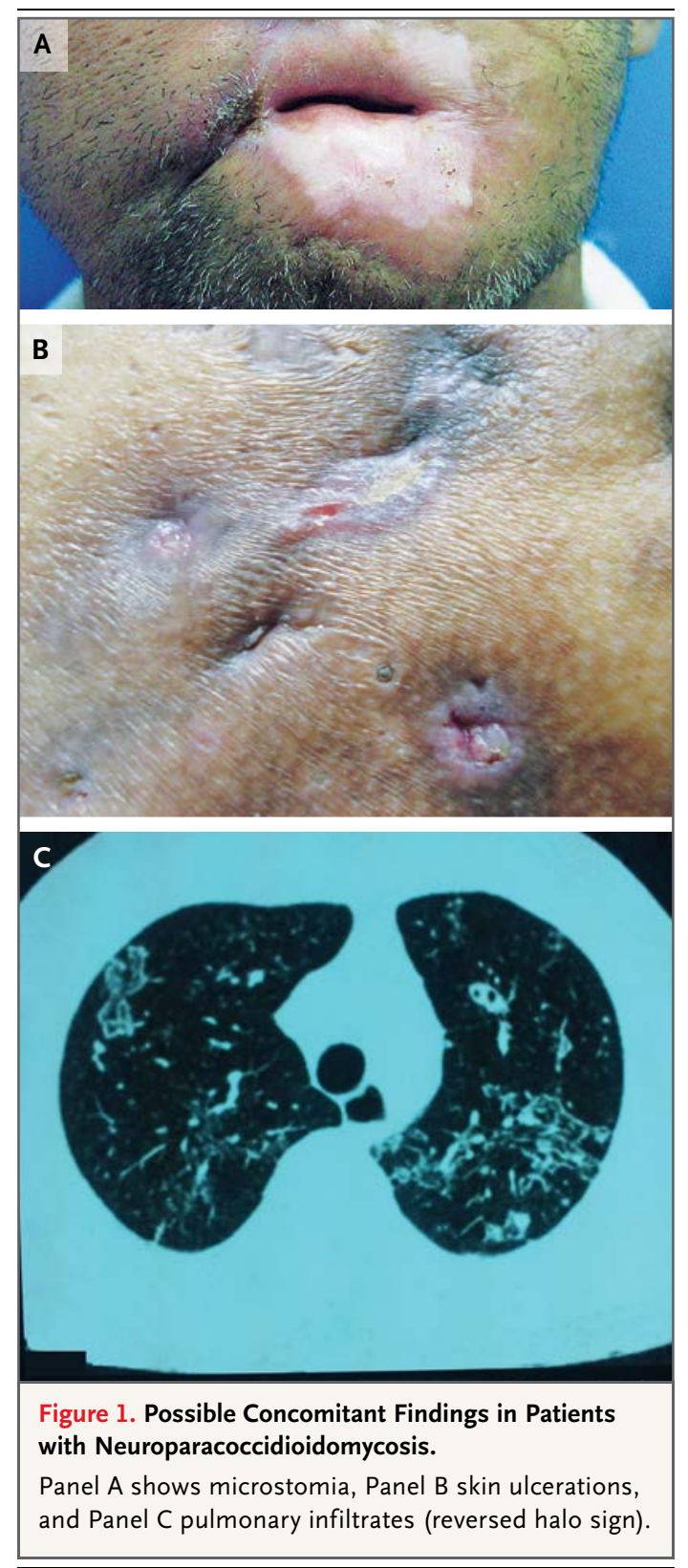

in those with concomitant oral lesions, microstomia, skin ulcerations, diffuse pulmonary infiltrates, or adrenal abnormalities (Fig. 1)..$^{1,3,4}$ This high clinical suspicion may allow early diagnosis and treatment, possibly reducing mortality and sequelae.

\section{Raquel M.N. Carvalho, M.D.}

Hospital Regional da Asa Norte

Brasilia, Brazil

\section{Samuel M. Nunes, D.D.S.}

Hospital Regional Público do Araguaia

Redenção, Brazil 
Alfredo N.C. Santana, M.D., Ph.D.

Escola Superior de Ciências da Saúde

Brasilia, Brazil

alfredoncs@gmail.com

No potential conflict of interest relevant to this letter was reported.

1. Shikanai-Yasuda MA, Telles Filho FdeQ, Mendes RP, Colombo AL, Moretti ML. Guidelines in paracoccidioidomycosis. Rev Soc Bras Med Trop 2006;39:297-310. (In Portuguese.)

2. Buitrago MJ, Bernal-Martínez L, Castelli MV, RodríguezTudela JL, Cuenca-Estrella M. Histoplasmosis and paracoccidioidomycosis in a non-endemic area: a review of cases and diagnosis. J Travel Med 2011;18:26-33.

3. Pedroso VS, Vilela MC, Pedroso ER, Teixeira AL. Paracoccidioidomycosis compromising the central nervous system: a systematic review of the literature. Rev Soc Bras Med Trop 2009; 42:691-7. (In Portuguese.)

4. Salaki JS, Louria DB, Chmel H. Fungal and yeast infections of the central nervous system: a clinical review. Medicine (Baltimore) 1984;63:108-32.

DOI: 10.1056/NEJMc1410501

THE AUTHORS REPLY: We agree with the concern regarding vancomycin penetration into the CSF, although a study has shown that the administration of high doses of intravenous vancomycin (60 mg per kilogram per day as a continuous infusion, after a loading dose of $15 \mathrm{mg}$ per kilogram), even with the concomitant administration of dexamethasone, in patients with bacterial meningitis led to adequate CSF concentrations (7.9 $\mu \mathrm{g}$ per milliliter), ${ }^{1}$ suggesting that the decreased penetration can be overcome with the administration of appropriate parenteral doses.

With regard to the penetration of vancomycin into brain abscesses, there are limited data. In one case report in a patient with an S. aureus-associated brain abscess, simultaneous measurements of vancomycin concentrations in serum and brainabscess fluid were obtained 1 hour after the intravenous administration of a 500-mg dose. Vancomycin levels obtained from the brain-abscess fluid before and during operative removal of the abscess were $15 \mu \mathrm{g}$ per milliliter and $18 \mu \mathrm{g}$ per milliliter, respectively; the serum vancomycin level was $21 \mu \mathrm{g}$ per milliliter. ${ }^{2}$ Pending further data, we continue to recommend treatment of brain abscesses caused by MRSA with vancomycin. However, careful monitoring of these patients is critical, and we agree that salvage therapy with relatively new agents (such as linezolid or daptomycin) should be used in patients with brain abscesses caused by MRSA strains who do not have a response or who have an elevated minimum inhibitory concentration of vancomycin.
The advised standard four-drug treatment regimen (isoniazid, rifampin, pyrazinamide, and ethambutol) for tuberculous brain abscesses is recommended in the current World Health Organization guidelines for tuberculosis of the central nervous system. Despite the excellent penetration of moxifloxacin into the CSF, a survival benefit with that drug in tuberculous meningitis has not been shown. ${ }^{3}$ Levofloxacin is currently being evaluated in a randomized, controlled trial involving patients with tuberculous meningitis. ${ }^{4}$ If this study shows benefits with levofloxacin, it would seem prudent to incorporate this agent into the treatment regimen for tuberculous brain abscesses.

In a recent meta-analysis that included 9699 patients with brain abscess, fungi comprised only a minority of cases (1.4\%), ${ }^{5}$ and paracoccidioidomycosis was rarely reported as an etiologic agent. However, we agree that neuroparacoccidioidomycosis should be strongly considered, in the appropriate clinical setting, as a cause of brain abscess in inhabitants of, and travelers from, regions in which this disease is endemic.

Matthijs C. Brouwer, M.D., Ph.D.

Academic Medical Center

Amsterdam, the Netherlands

Allan R. Tunkel, M.D., Ph.D.

Warren Alpert Medical School of Brown University Providence, RI

Diederik van de Beek, M.D., Ph.D.

Academic Medical Center

Amsterdam, the Netherlands

d.vandebeek@amc.uva.nl

Since publication of their article, the authors report no further potential conflict of interest.

1. Ricard JD, Wolff M, Lacherade JC, et al. Levels of vancomycin in cerebrospinal fluid of adult patients receiving adjunctive corticosteroids to treat pneumococcal meningitis: a prospective multicenter observational study. Clin Infect Dis 2007;44:250-5.

2. Levy RM, Gutin PH, Baskin DS, Pons VG. Vancomycin penetration of a brain abscess: case report and review of the literature. Neurosurgery 1986;18:632-6.

3. Ruslami $R$, Ganiem $A R$, Dian $S$, et al. Intensified regimen containing rifampicin and moxifloxacin for tuberculous meningitis: an open-label, randomised controlled phase 2 trial. Lancet Infect Dis 2013;13:27-35.

4. Heemskerk D, Day J, Chau TT, et al. Intensified treatment with high dose rifampicin and levofloxacin compared to standard treatment for adult patients with tuberculous meningitis (TBM-IT): protocol for a randomized controlled trial. Trials 2011;12:25.

5. Brouwer MC, Coutinho JM, van de Beek D. Clinical characteristics and outcome of brain abscess: systematic review and meta-analysis. Neurology 2014;82:806-13.

DOI: 10.1056/NEJMc1410501

Correspondence Copyright (๑) 2014 Massachusetts Medical Society. 